«Éprouver pour savoir » : l'observation participante d'Elizabeth L. Banks

'Learning through Experience': Elizabeth L. Banks as Participant Observer

\title{
Nathalie Saudo-Welby
}

\section{(2) OpenEdition}

1 Journals

Édition électronique

URL : http://journals.openedition.org/rfcb/6127

ISSN : 2429-4373

Éditeur

CRECIB - Centre de recherche et d'études en civilisation britannique

Édition imprimée

Date de publication : 1 juin 2010

ISSN : 0248-9015

\section{Référence électronique}

Nathalie Saudo-Welby, « «Éprouver pour savoir » : l'observation participante d'Elizabeth L. Banks »,

Revue Française de Civilisation Britannique [En ligne], XV-4 | 2010, mis en ligne le 01 juin 2010, consulté le 07 janvier 2021. URL : http://journals.openedition.org/rfcb/6127

Ce document a été généré automatiquement le 7 janvier 2021.

\section{(†) $\odot$

Revue française de civilisation britannique est mis à disposition selon les termes de la licence Creative Commons Attribution - Pas d'Utilisation Commerciale - Pas de Modification 4.0 International. 


\title{
«Éprouver pour savoir»: l'observation participante d'Elizabeth L. Banks
}

\author{
'Learning through Experience': Elizabeth L. Banks as Participant Observer
}

\author{
Nathalie Saudo-Welby
}

1 Dans une série d'articles parus dans la revue The Nineteenth Century et publiés en 1894 sous le titre Campaigns of Curiosity: Journalistic Adventures of an American Girl in Victorian England, Elizabeth L. Banks se livra à une série d'enquêtes sur le rapport entre les femmes, leur activité et l'argent. Pour les écrire, elle se fit successivement passer pour une servante ('In Cap and Apron'), une riche héritière américaine en quête d'un chaperon ('The Almighty Dollar in London Society'), une Américaine en quête d'ancêtres ('The Price of a Pedigree'), une balayeuse de rue ('Sweeping a Crossing'), une vendeuse de fleurs ('A Day with the Flower-Girls') et une blanchisseuse ('Among the Laundry-Girls'). En se livrant à ces mascarades, elle s'inscrivait dans la tradition du journalisme à sensation ('yellow journalism') et du journalisme d'immersion ('stunt journalism'), dans lesquelles Nellie Bly s'était illustrée. Bly est demeurée célèbre pour s'être fait passer pour folle et enfermer à l'asile pour femmes de Blackwell's Island, une aventure ensuite publiée sous le titre Ten Days in a Mad-House (1887) et pour avoir fait le tour du monde en 72 jours, une aventure racontée dans le New York World en 1889-90. Ces pratiques suscitaient le mépris parce que leur succès reposait sur une forme de voyeurisme qui avait d'autant plus de succès qu'il était spectaculaire, mais également parce que leurs auteurs étaient souvent peu soucieux des impératifs éthiques de leur profession ${ }^{1}$.

2 Aujourd'hui, les écrits de Banks peuvent inspirer autant de mépris de la part de ceux qui y chercheraient des documents sociologiques ou des textes dits «de civilisation ». Campaigns of Curiosity d'Elizabeth Banks fait en effet partie des œuvres difficiles à classer $^{2}$ : récit de voyage, autobiographie, journalisme à sensation, enquête sociale? Republié sous la forme d'un fac-similé par les Presses de l'Université du Wisconsin dans la section des autobiographies, l'ouvrage, qui soulève les questions de la sincérité et de 
la fiabilité inséparables du genre, se présente comme une étrange forme d'autobiographie puisque Banks raconte ses propres expériences dans le but de parler de la vie des autres. Elle introduit par ce biais de multiples filtres, ce qui résulte en une imbrication de nombreux niveaux de représentation: son jeu d'actrice dans ses différentes incarnations, sa nature de petite-bourgeoise américaine qu'elle ne tenta jamais de dissimuler ${ }^{3}$, et sa propre tâche d'écriture, doublement contrainte par les exigences de l'autobiographie et de la presse à grand tirage. Les expériences de Banks nous renseignent sur l'accès pour une femme à la profession de journaliste dans les années 1890, mais proposent-elles des éléments d'analyse des professions féminines qu'elle décrit?

3 Ces pages ont pour objectif, grâce aux outils théoriques développés aujourd'hui par les praticiens de l'observation participante, d'analyser un texte d'apparence lisse et simple, qui raconte à son niveau le plus évident la percée d'une femme résolue. On cherchera par une analyse des différents niveaux de représentations à comprendre comment l'écriture de Campaigns of Curiosity s'intègre dans une démarche de connaissance et à examiner comment la subjectivité d'Elizabeth Banks permet de mieux décrire la condition des femmes de son temps.

Banks n'a jamais caché que les raisons qui gouvernaient ces expériences étaient financières et professionnelles et non cognitives et culturelles. Dans son Autobiography of a Newspaper Girl (1902), elle a ouvertement déclaré qu'en s'intéressant à la condition de personnes étrangères, elle n'avait fait que suivre son propre intérêt : négocier son entrée dans le monde du journalisme, et gagner suffisamment pour ne pas mourir de faim. La longueur de ses séjours d'immersion, qui s'étend d'une semaine dans le cas du service de maison à quelques heures dans le cas de la recherche d'un chaperon, obéissait à des critères peu scientifiques. Il s'agissait d'avoir collecté suffisamment d'impressions et de matière pour écrire un article vendeur. Néanmoins, cela ne peut suffire à mettre en cause le sérieux de son entreprise car dans la majorité des cas, elle était soucieuse de renouveler la même expérience auprès d'employeurs différents afin de changer de perspective.

5 Banks n'est pas une de celles qui tentèrent de s'immerger dans la vie de leurs objets d'étude à des fins sociologiques. Le but avoué de gagner de l'argent par ces mascarades pourrait affaiblir la dimension cognitive de son expérience, mais il la rapproche aussi des personnes qu'elle tente d'incarner. Banks insiste à de nombreuses reprises sur la proximité que sa condition financière créait avec les femmes qu'elle étudiait, mais elle redoutait de publier trop ouvertement sa véritable condition de crainte d'aliéner une partie de l'opinion, fatiguée par les multiples formes prises par le discours philanthropique :

I reasoned it out that if London knew that the amateur housemaid who essayed to put her experiences into print was quite as much compelled by necessity to go into domestic service in order to earn her living as was any real housemaid who ever applied to a London mistress for a situation, then London might not be so much interested in the story of my ups and downs, on the principle that the poor it had ever with it, and was rather longing for a change. (ANG 85)

Son choix se porta ainsi sur une forme qui excusait l'intrusion du désagréable sous des codes plaisants: le récit de voyage. Les multiples métamorphoses de Banks et Bly étaient racontées sous une forme comique et pittoresque. L'un des ressorts comiques des récits de Banks provient du rapprochement créé entre son séjour parmi les servantes et une expédition ethnologique chez une peuplade de sauvages : il lui faut 
emporter des vivres, elle rencontre des difficultés à communiquer et transcrit avec soin l'accent cockney de ses collègues. Bien que son entreprise soit fondée sur l'idée d'immersion, elle répugne à vivre l'expérience dans sa totalité, comme lorsqu'elle précise dans son annonce qu'elle ne sera pas prête à partager un lit avec ses collègues ${ }^{4}$, détail comique susceptible de provoquer chez les lectrices un petit frisson d'effroi.

Dans la préface qui accompagna la publication des articles, Banks attribua à ses facéties une fonction plus sérieuse. Elle concéda que son travail au milieu des servantes et des blanchisseuses avait affecté la façon dont ces professions étaient perçues et même influencé les réformes législatives ${ }^{5}$. Mais en traitant le reste de ses expériences comme de vulgaires mascarades, elle encouragea le reste de l'opinion à considérer ses articles comme 'a journalistic sowing of wild oats' (CC xvi): 'the two articles which recount my experiences as a flower-girl and a crossing-sweeper [...] do not take up any very serious social or moral problems.' (CC xiv).

\section{Mise en scène de soi}

Dans le chapitre consacré à Elizabeth Banks dans son ouvrage Slumming: Sexual and Social Politics in Victorian London, l'historien Seth Koven semble ne lui accorder que bien malgré lui une fonction sociologique. Au regard de Margaret Harkness, qui sut, selon lui, séparer fiction et documentation et se livrer à ses heures à une recherche objective $^{6}$, Elizabeth Banks est coupable d'égocentrisme.

Comme l'indique le titre de son ouvrage, Campaigns of Curiosity: Journalistic Adventures of an American Girl in Late Victorian London, Elizabeth Banks se met au centre des enquêtes. Le volume s'ouvre d'ailleurs sur un portrait de l'auteur. Son sous-titre affiche ouvertement son identité de femme américaine comme si cette identité constituait en soi une valeur ajoutée. En d'autres termes, Banks présente l'originalité du livre comme émanant du point de vue de l'auteur et non de l'objet d'étude lui-même. Les illustrations sont à cet égard exemplaires : elles n'ont aucune fonction documentaire mais font surtout vibrer la corde sentimentale. Des photographies de l'auteur en costume pittoresque, prises en studio, soulignent le charme inaltérable et la vulnérabilité de l'héroine. Les autres illustrations ressemblent aux gravures insérées comme des petites vignettes dans un roman; elles illustrent les grands moments comiques des aventures, comme l'ouverture d'une bouteille de vin (CC 69) ou 'My system of scrubbing' (CC 56). La seule exception est une gravure représentant la figure de Janie, une jeune blanchisseuse boiteuse pleine d'égards pour Banks qui se prit d'une véritable sympathie pour elle.

10 Selon la façon dont on choisit d'aborder ses textes, leur subjectivité peut faire figure d'un obstacle à la connaissance ou d'une médiation salutaire. La connaissance qu'a Banks de son sujet est-elle limitée ou au contraire amplifiée par sa propre subjectivité ? L'enquête de Banks prend la forme d'une mise en scène, elle-même présentée dans un texte. Toute représentation est dans ce cadre ce que les Anglo-Saxons appellent une 'misrepresentation' : elle sera partiale, sélective et subjective, guidée par des impératifs bien éloignés de la sociologie, comme la nécessité de plaire à son lectorat, articulée à la nécessité de gagner un salaire, le désir de faire bonne figure (Banks était particulièrement soucieuse de ne pas sacrifier sa féminité et sa moralité à son activité de journaliste), sa conscience de classe et l'affirmation de son identité américaine. Dans son autobiographie, Banks définit clairement son activité comme une forme de mise- 
en-scène de soi $(A N G 75,86)$ et reconnut même volontiers à plusieurs occasions avoir surjoué le rôle qui lui incombait :

Nearly all of the tasks I was given to perform I did well and conscientiously. I say nearly all, for there were certain kinds of work I thought it well to attempt in peculiar and original ways, in order to 'draw out' observations from my fellowservants and occasionally to note the effect upon my mistress. I did not allow myself for one instant to forget that I was a journalist seeking 'copy,' and I had no notion of letting any opportunities for getting that all-important article slip by me.

(ANG 81)

11 Dans ces moments-là, Banks n'est ni elle-même ni celle qu'elle est censée incarner. Elle est en double situation d'imposture, comme servante et comme godiche. Les impératifs de l'écriture la conduisent à déformer son expérience, à prendre la pose : 'I also did what might possibly be termed a little 'posing' during the course of my narrative, by letting it appear that I was not well up in the art of housework.' (ANG 86) Son activité d'écriture s'inscrit donc dans la continuité de son activité d'actrice sur le terrain.

12 Au-delà des nombreux rôles socio-professionnels énumérés ci-dessus, lesquels nécessitaient un déguisement et des formes d'introduction particulières, Banks a ainsi joué de multiples rôles : la femme célibataire soucieuse de se distinguer des 'hysterical sisters'; l'Américaine soucieuse de donner une bonne image à une époque où la femme anglaise et la femme américaine faisaient l'objet d'analyses comparées dans les journaux ; la femme-journaliste en herbe, un rôle encore suffisamment mal établi pour qu'il soit nécessaire de le créer. Comme nombre de Victoriens, ${ }^{7}$ Banks semblait ainsi engagée dans une activité de constante représentation de soi dans des rôles qui mettaient en valeur son extraordinaire personnalité.

\section{Observation participante}

13 À l'exception de son étude des blanchisseuses, qui se rapproche le plus de la sociologie traditionnelle, les enquêtes de Banks, qui contiennent peu de chiffres et de statistiques, reposent sur une expérience personnelle de la mascarade et de l'imposture. À ceux qui reprochent à ses aventures de n'être qu'une mise en scène trahissant son narcissisme, il est possible de répondre que le texte demeure comme un témoignage intéressant sur des expériences professionnelles rarement racontées par leurs sujets. L'histoire sociale ne peut se passer de la fiction pour reconstruire la culture et les mentalités de la fin du $\mathrm{XIX}^{\mathrm{e}}$ siècle ${ }^{8}$.

14 Une réponse plus fructueuse consiste à postuler que le décentrement réalisé au cours de ces expériences est en soi une démarche de connaissance. Sortir de soi et investir un objet physiquement pour le connaître de l'intérieur, en éprouvant parfois au-dedans de soi des vestiges de ses perceptions anciennes, telle est la démarche de Banks. L'intérêt de ses récits d'aventure repose sur l'ambiguïté qui se crée entre la mise en scène de soi et la capacité à se décentrer. Banks joue-t-elle un rôle ? Se met-elle à la place d'autrui ? Dans le premier cas, les aventures mettraient en valeur ses talents d'actrices et opéreraient une mise en valeur de soi. Dans le deuxième, ses aventures révèleraient une véritable empathie capable d'opérer un bouleversement des valeurs. Banks se met en scène elle-même afin de donner à voir quelque chose qui lui est étranger, ce qui est une façon d'accueillir l'autre en soi. L'égocentrisme de la démarche et les raisons qui la motivent apparaissent sous un jour nouveau si on fait le choix d'envisager les textes de Banks à l'intérieur de la naissance d'une certaine forme de sociologie qualitative. Par le 
choix répété d'enquêter en s'immergeant dans le milieu d'étude ${ }^{9}$, Elizabeth Banks exprimait clairement sa conviction que son objet ne pouvait être bien étudié que de l'intérieur, à partir d'une expérience subjective: 'As a mistress, however kind and considerate I might be, it was impossible for me to get a perfect understanding of the inner work of the household machinery. There was only one way to get at the root of the matter, that was to go out to service myself.' (CC 6) L'attitude de Banks consiste ainsi à remettre en question ses propres représentations par la confrontation directe avec ce qu'elle cherche à connaître. Elle est à la fois observatrice et participante.

L'observation participante est une des formes de la recherche qualitative en sciences sociales (ethnologie, anthropologie et sociologie). Elle consiste à interagir depuis l'intérieur avec le milieu que l'on souhaite étudier. Les observateurs participants peuvent être plus ou moins déclarés, allant du participant complet, qui dissimule son véritable objectif, tel un espion, à l'observateur complet dont la présence est ignorée de ses sujets d'étude ${ }^{10}$. À ses sources, avant qu'elle ne soit théorisée et pratiquée par l'Ecole de Chicago dans les années 1920, l'observation participante est l'invention des écrivains (les romanciers réalistes) et des journalistes d'investigation ${ }^{11}$.

Dans un ouvrage de synthèse sur cette pratique, Jean Copans écrit de ses productions qu'elles sont «écriture » pour rendre compte de l'important effort mis en œuvre dans l'élaboration du compte-rendu. L'observation participante, activité pratique, s'inscrit donc, aux deux bouts de la chaîne, dans l'écrit: des notes de terrain au texte. "L'ethnologie en devenant texte réduit les pratiques de l'enquête à de simples pré-textes: le lecteur qui souhaite toucher le réel du doigt rencontre ainsi une difficulté supplémentaire. Il faut qu'il se hisse sur la pointe des pieds pour essayer de lire directement le terrain par-dessus l'épaule de l'ethnologue devenu écrivain. $»^{12}$

Invention d'écrivains devenue pratique d'écriture, l'observation participante se trouve être presque indissociable d'une activité littéraire. Elle raconte une histoire, qui inclut des moments de crise et des péripéties. Jean Copans distingue trois grandes étapes du séjour de longue durée : l'arrivée et l'installation, l'intégration et la routine, l'imprévu et la crise. Les théoriciens de l'observation participante insistent sur l'intérêt sociologique de toute la démarche: négociation de l'accès au terrain, installation, expériences et négociation de la sortie. L'observation participante est aussi une activité dramatique qui nécessite du talent d'acteur, des costumes et du maquillage ${ }^{13}$. Enfin, c'est une expérience subjective proche de l'autobiographie, où le sujet explorant, pour parler des autres, se raconte lui-même. Cette activité est créative : elle consiste, en agissant et en pensant avec et à la place de, à questionner ses représentations pour proposer des alternatives. L'observation participante est une activité de décentrement.

Dans les pages qui suivent, je vais tenter d'analyser les expériences de Banks telles qu'elles sont présentées dans deux de ses œuvres (Campaigns of Curiosity et Autobiography of a Newspaper Girl) à partir de quelques notions clés de la méthode sociologique appelée "observation participante » : le décentrement, la négociation de l'accès au terrain, la médiation du corps et la mise en écriture.

\section{Décentrement}

19 On ne peut qu'être impressionné par le nombre de femmes qui se sont, même assez tôt, adonnées à l'observation participante. Jean Péneff donne des exemples des générations de l'après-guerre: Simone Weil, Jacqueline Frisch-Gauthier, Véronique Linhart, 
Gwenaële Rot ${ }^{14}$. Il donne aussi des exemples récents d'« entrisme journalistique » : M. Riffaud se faisant aide-soignante, Laurence Lacour et l'affaire "Grégory ", Florence Aubenas et le procès d'Outreau et Florence Beaugé en Algérie ${ }^{15}$. Malgré tous ces cas, Jean Péneff ne mène pas de réflexion particulière sur ce qui pourrait encourager les femmes à se livrer à ce type d'enquêtes par voies détournées. Au contraire, concluant son chapitre sur «Romanciers, Journalistes et Artistes " avec l'exemple d'Isabelle Eberhardt (1877-1904) qui s'immergea parmi les bédouins et en rapporta une excellente connaissance de la société nomade, il rapporte sans les commenter les propos de sa biographe, Edmonde Charles-Roux: "Vêtue en cavalier et couchée dans un coin, on ne remarquait pas Isabelle. Excellente position pour bien voir, notait-elle. Si les femmes ne sont pas de grandes observatrices, c'est que leur costume attire les regards, elles ont toujours été faites pour être regardées. ${ }^{16}$ Peneff cite aussi ces conseils de Colette qui suggèrent que la femme a davantage d'efforts à fournir pour observer et s'identifier, qu'elle est plus difficile à « décentrer »: "Nous ne regardons, nous ne regarderons jamais assez, jamais assez juste, jamais assez passionnément ! ${ }^{17}$.

Dans Storytelling Sociology: Narrative as Social Inquiry, Ronald J. Berger and Richard Quinney rendent au contraire les féministes responsables de l'évolution de la sociologie vers la recherche qualitative :

Feminism in particular played a key role in debunking sociology's privileged knowledge claims. Feminist scholars argued that 'truth' is contested and polyvocal and that women's social position in society gives them distinctive insights, indeed a different epistemological standpoint, from men's. They advocated a research methodology that would eschew personal detachment and encourage collaboration and empathic connection with research subjects, cross-fertilization among academic disiciplines, and involvement in action-oriented research that would facilitate personal and social transformation. ${ }^{18}$

21 On peut observer dans ce passage la façon dont des qualités traditionnellement associées aux femmes sont placées au centre de la recherche qualitative : la capacité d'empathie ('empathic connection with research subjects') et la volonté de se mettre en scène et de jouer un rôle ('personal and social transformation'). Comme le montre Seth Koven dans son analyse, Elizabeth Banks essaya à tout prix de se distinguer des démarches des philanthropes qui, sous prétexte d'empathie, trouvaient plaisir à une activité tellement répandue et institutionnalisée qu'elle avait pris un nom : 'slumming'19.

Dans le premier des trois passages que je viens de citer, la femme est représentée par un passif ("être regardées »); dans le deuxième, Colette utilise une injonction qui, couplée à une négation, semble déboucher sur un perpétuel échec; dans le dernier, les femmes, devenues des 'scholars', sont placées en position de décideuses ('argued', 'advocated'). Le décentrement permis à la femme me semble résulter de la conscience de cette évolution: la perception affinée par leur propre expérience sociale, les sociologues féministes décrites ci-dessus tirent profit des positions qui leur sont traditionnellement attribuées pour tenir un propos "décentré ». De même, en se mettant en scène, Elizabeth Banks joue conjointement de sa position de femme-objet, destinée à être regardée, et du recul que lui donne sa riche identité. Cette identité est plurielle : pauvre, et donc amenée à travailler pour vivre sur le marché du travail ; femme convoitant une profession masculine ; Américaine observant l'Angleterre avec des yeux d"outsider'. Dotée d'une identité forte, Banks se décrit aussi comme très capable de l'évacuer. Elle dit avoir été presque progressivement gagnée par l'accent cockney (CC 172). Elle rapporte également cet éloge d'un de ses éditeurs prêt à 
l'envoyer en mission délicate : 'You've got diplomacy, tact, shrewdness, discretion, and, above all, you are absolutely feminine, and you haven't got "newspaper woman" and "interviewer" placarded all over you.' (ANG 253) Malgré son identité très riche et sa féminité, qu'elle défend contre toutes les dérives de la 'New Woman', Banks a toutes les qualités d'un espion potentiel. La préface raconte comment après la publication de sa première expérience de servante, elle passa pour leur défendeuse, alors qu'elle fut à la publication de sa seconde aventure considérée comme une infâme traitresse (CC x-xi). Espionne pour la classe qu'elle représente ('Oh, you! You're nothin' but a spy and houtsider, any'ow. You ain't no proper servant.' ANG 83), traîtresse pour la classe qu'elle incarne momentanément, réformatrice en tant que journaliste ('doing evil that good may come'), Banks joue tous les rôles à la fois ${ }^{20}$.

Dans la mesure où ses intérêts sont engagés et partagés, Banks ne prétend nullement à une écriture objective. Le jeu de rôles est une mise en scène de soi qui ne débouche pas sur une mise en valeur de soi ; il s'agit de renoncer à son rôle social tout en s'éveillant à l'appréciation des différences. Son style, qui joue des différentes formes de distanciations permises par l'écriture, montre son art à se décentrer. La finesse d'appréciation de Banks, qui est venue collecter non pas des faits mais des paroles et des impressions, apparaît dans ses mensonges ouverts ou couverts, dans ses dialogues retranscrits avec des transpositions phonétiques, dans son ironie tantôt franche tantôt flottante s'exerçant à l'encontre de l'un, de l'autre puis d'elle-même.

Sa très grande conscience de classe ne la quitte jamais et lui permet d'être particulièrement sensible aux termes d'adresse employés. Elle observe ainsi qu'elle est devenue 'a young person', jongle habilement avec les termes 'girl', 'woman' et 'lady' (CC 5) et observe comment les rôles sont à l'occasion inversés lorsqu'une jeune bourgeoise vient servir les blanchisseuses à table (CC 198). Mais cette conscience lui permet de délibérément renverser les rôles: 'They are grateful to anyone who really tries to help them in the right way, which is by putting oneself as much as possible on a level with them and not attempting to show any superiority.' (CC 207) Banks n'a aucun mal à se plier à cette contrainte car son expérience l'a rendue l'une des leurs, même si les blanchisseuses ont pour elle le respect dû à une 'lady' (CC 200) : 'The fact that I had done laundry-work, no matter for what purpose, put me in their good graces at once, and they treated me as one of themselves, inviting me to become a member of the club by the payment of fourpence a month, the fee required of each member' (CC 197-98).

Les enquêtes de Banks ne dérivent pas du désir de placer son lecteur en position de voyeur de scènes titillantes ou de faire de lui un transgresseur en des lieux inaccessibles ${ }^{21}$. Quel lecteur de The Nineteenth Century n'avait pas lui-même fait l'expérience de rapports avec des domestiques? La presse de l'époque n'était-elle pas remplie d'avis sur 'The Servant Problem', question sur laquelle chacun prétendait pouvoir légitimement se prononcer? N'aurait-il pas suffi à chacun de sortir de chez lui pour observer la vendeuse de fleurs ou la balayeuse de rue? La démarche de Banks visait bien à décentrer le lecteur en lui faisant observer de l'intérieur des situations familières. Partie en Angleterre pour écrire un livre d'amusantes observations à l'intention de ses compatriotes (CC ix), Banks écrivit finalement un ouvrage visant à faire découvrir aux Anglais des scènes familières !

Sa première enquête naquit d'un étonnement. Souhaitant venir en aide à sa couturière, elle lui proposa de la prendre à son service. Devant la réaction indignée de celle-ci, Banks s'étonna: pourquoi tant de femmes rechignaient-elles à cette occupation et 
brandissaient-elles avec détermination le mot d'« indépendance »? Cet étonnement conduisit Banks à franchir toutes les étapes la séparant de cette profession afin de comprendre comment l'indépendance de ces femmes se trouvait menacée. De fait, les enquêtes de Banks naissent toujours d'un questionnement sur un cliché ou un préjugé qu'elle souhaite examiner. Son enquête sur la question des chaperones prit sa source dans l'affirmation d'un Anglais qui soutenait que l'aristocratie anglaise se définissait par des valeurs où l'argent n'entrait pas. C'est en souvenir des paniques hygiéniques auxquelles la classe moyenne américaine cédait régulièrement qu'elle prit le parti de suivre son propre linge jusqu'à la blanchisserie, allant jusqu'à le marquer elle-même. Par ce biais, elle s'assura aussi de la fausseté du préjugé qui fait des blanchisseuses de violentes poissardes à la moralité douteuse.

Le chapitre sur les balayeurs de rue s'ouvre sur une série de légendes faisant d'eux des hommes riches et sophistiqués (CC 130). Banks avait probablement en tête deux articles publiés dans Tit-Bits ('A Day as a Professional Beggar' 17 janvier 1891 et 'A Day as an Amateur Crossing Sweeper' 18 avril 1891). Leur auteur s'était fait passer pour un mendiant et un balayeur et avait ainsi fait courir la rumeur qu'un mendiant 'professionnel' et artiste pouvait gagner 3 shillings et 6 pence, et un balayeur un shilling et trois pence. Il faut observer que Arthur Conan Doyle, lui-même journaliste, a écrit une nouvelle qui confirmait l'idée que les mendiants n'étaient qu'un résidu parasite qui vit aux dépens des Londoniens. Dans 'The Man with the Twisted Lip' (1891), le journaliste Neville St Clair ayant découvert en faisant de l'observation participante que les mendiants pouvaient gagner plus de 25 shillings en sept heures de temps, il se reconvertit dans cette activité moins fatiguante et plus lucrative ${ }^{22}$. Une fois la supercherie découverte, Sherlock Holmes va agir pour remettre de l'ordre dans la classe moyenne et renvoyer cet homme au travail. Rentrée chez elle avec trois malheureux pennies, Banks disqualifie entièrement ces mythes tenaces. Sa compassion pour les balayeurs est immense, et, comme dans le cas des vendeuses de fleurs, elle ira jusqu'à excuser leur manque de sincérité et de probité, qu'elle compare presque explicitement à ses propres manquements éthiques en tant que journaliste. Les mensonges attendrissants racontés par les balayeurs sont décrits dans les mêmes termes que sa propre activité de journalisme comme 'doing evil that good might come' (CC 19, 138).

Dans son analyse des vendeuses de fleurs londoniennes, Banks affiche tout au long de son article des préjugés tenaces et profondément ancrés : elles sont violentes, vulgaires et malhonnêtes. Banks constate que, par la force du sang et l'exemple, ces femmes, vendeuses de fleurs de mère en fille, se suivent et se ressemblent. Ses préjugés ne seront pas démentis. Mais son histoire a la force d'une argumentation : tandis qu'elle s'est efforcée d'incarner une figure opposée, faite de grâce, d'humilité et de douceur, composant son panier avec art et apitoyant les passants sur son honnêteté méritante, elle découvre avec surprise en comptant ses maigres bénéfices que son interprétation du rôle n'a pas payé: elle a gagné deux fois moins que le revenu moyen de ses comparses! 'I am in a quandary as to how the thing is done.' (ANG 151) Banks se garde de conclure mais le lecteur est contraint d'en déduire que la démoralisation de ses alteregos n'est qu'une conséquence de leur faible niveau de revenus et une stratégie nécessaire pour les augmenter. 


\section{La négociation de l'accès au terrain}

29 La plus grande partie des récits de Banks est consacrée à cette question, et ces pages contiennent les éléments les plus comiques en même temps que les plus révélateurs. La négociation de l'accès au terrain n'est pas antérieure à l'observation, elle en est une première étape :

Et cette négociation, en tant qu'elle comporte déjà des interactions intenses avec les gens, est l'occasion d'effectuer les premières observations ; elle fait donc déjà partie de l'observation participante. Rien, d'ailleurs, n'est jamais acquis définitivement, il faudra toujours, et jusqu'au bout de la recherche engagée, re-négocier l'entrée. On peut considérer à cet égard que le travail consistant à analyser en permanence l'institution - au sens actif du terme - de cette recherche en constitue une dimension essentielle. ${ }^{23}$

Qu'elle joue la servante ou la riche héritière américaine, les enquêtes de Banks commencent de la même façon: elle rédige une annonce et la fait paraître dans un quotidien britannique, puis attend chez elle les réponses. Ménageant de grands effets de surprise et des effets comiques d'accumulation, Banks montre comment, ayant fait paraître une annonce pour un emploi de servante dans un quotidien britannique, elle fut récompensée par 159 réponses. Ayant sélectionné les seules propositions qui correspondaient à ses attentes, elle répondit et se rendit à des entretiens. Mais pensant faire acte d'humanité en se mettant au service au lieu de se faire servir, Banks découvre que cette place ne lui est pas facilement accordée et va essuyer de nombreux refus. Banks peine à se faire accepter parce qu'elle porte une frange, parce qu'elle est trop petite, pas assez costaude, puis trop effrontée. Banks se trouve confrontée aux préjugés de sa propre classe ${ }^{24}$ et le lecteur fait l'expérience du sectarisme ambiant, des préjugés et de la discrimination.

31 En rapprochant cette enquête de celle qu'elle mena pour explorer le marché des chaperones, il apparaît qu'il est plus aisé de se faire passer pour une riche héritière américaine que pour une servante. Dans ce rôle qu'elle n'eut aucun mal à jouer avec l'aide de sa propre couturière, Banks ne courut aucun risque d'être démasquée. La société britannique décrite par Banks est composée d'un ensemble de places à négocier, et le rôle le plus facile à jouer et le plus ouvert, pour qui a l'argent, est de se faire introduire dans la bonne société anglaise et obtenir une introduction à la cour d'Angleterre. Par un effet d'enchâssement, Banks doit pour négocier son accès à la profession de journaliste se faire passer pour une riche héritière qui tente elle-même de se faire une place dans la bonne société britannique par des voies détournées.

Outre le potentiel comique des parallèles de situation, il faut voir dans la structure qui force Elizabeth Banks à se faire accepter comme servante, puis blanchisseuse, puis balayeuse de rue pour se faire accepter comme journaliste, un effet de mise en abyme intéressant. Banks reproduit ses propres tentatives malheureuses d'obtenir une place de sténographe dans tous les journaux américains connus d'elle. Son expérience malheureuse est relatée en ces termes :

Never an answer came back, though I had enclosed a stamped envelope for reply in every letter. I decided that editors must be approached personally, and not by letter, and I concluded that in order to get to the places where editors lived and newspapers were published, I must obtain some sort of situation in a large town. Then I sent out another batch of letters, this time to all the large stores that I saw advertised in the semi-weekly newspaper that came to the farm, asking for a position as stenographer and typewriter at any wages they liked to offer. From my 
thirty letters I got one reply, offering me a situation. It was from a wholesale grocer in a large Western city, who offered me eight dollars a week to write his letters and keep account of his cash. The situation was accepted. Then came my second leavetaking at the village station, and then I went out into the world, confident, though all alone, committed to the charge of the angels. (ANG 3) envoyant à un éditeur le récit de son expérience sous le titre 'All about Typewriter Girls' que Banks fit son entrée dans la sphère du journalisme comme secrétaire, puis comme rédactrice. En publiant des morceaux de réalité de sa vie imparfaite et non désirée, Banks accéda à l'emploi désiré. Pour maintenir sa place dans cet univers, ses enquêtes allaient ensuite reproduire ce moment fondateur, où elle adressait à un éditeur son compte-rendu avec ces mots: 'Please publish this in your next Sunday's paper. It is all true.' (ANG 5) Les rôles envisagés par Banks lui permettent des moments de transgression passagers. Comme James Greenwood et Barnado, elle fait partie des nombreux 'slummers' de la période victorienne: 'many were deeply invested in the titillating squalor of the slums, which they used as stages upon which they enacted emancipatory experiments in reimagining themselves. ${ }^{25}$ Mais en se livrant à ses mascarades, Banks reproduit sa propre démarche de négociation d'une place de journaliste. Il est important que Banks se trouve dans la même situation financière et émotionnelle que la femme qu'elle représente. La nécessité qu'avait Banks, comme les jeunes femmes qu'elle imitait, de gagner sa vie ${ }^{26}$ lui permet de ne pas être partisane. L'enquête de Banks naît d'une curiosité vis-à-vis d'une position qu'elle pourrait envisager pour ellemême. Le lecteur contemporain ne peut qu'être sensible à la façon dont elle utilise cet argument pour excuser ses mensonges et ses trahisons à sa propre classe, mais aussi un certain manque d'empathie vis-à-vis de son sujet.

Cette structure littéraire de mise en abyme aboutit à faire du travail féminin quelque chose qui doit se conquérir, une condition difficile à atteindre quel que soit l'emploi convoité. En revanche, les deux ouvrages de Banks font sans cesse référence à un choix par défaut et jamais exploré : le mariage. Lorsqu'elle prétend être une jeune fille de bonne famille qui recherche un emploi de maison, elle reçoit une demande en mariage. La même proposition l'attend lorsqu'elle prétend être une riche héritière en quête de relations. Revenue aux Etats-Unis où elle écrit la chronique de sa vie avec «trois dollars par jour ", elle reçoit une honnête proposition d'un ouvrier qui n'a pas compris l'imposture (ANG 265). Elle reçoit le courrier enflammé d'un admirateur français amoureux de ses talents littéraires et de sa vivacité (ANG 270).

The young English mechanic desired to marry a housemaid; the young American aspired to the hand of a factory girl who was starving and freezing on three dollars a week, and the Englishmen of noble lineage paid their courtly devotions to a supposed Miss Money-bags, who had dollars to burn. I take no credit to myself on account of them. They were not intended for me, but for the person I misrepresented myself to be. (ANG 269)

Le mariage est systématiquement présenté comme un état qui lui permet d'échapper à sa situation. Devenue journaliste, sa vulnérabilité fait regretter à ses collègues, forcés de la raccompagner chez elle le soir, qu'elle n'ait pas fait un choix de vie plus convenable et plus commode, et Banks entend un de ses collègues s'écrier : 'I don't care how nice they are as girls, they're nothing but nuisances in a place like this at midnight. While I'm walking home with her I'll just ask the young lady to marry me, and that'll put an end to all our troubles!' (ANG 17) En se mettant en scène comme servante et comme chasseuse 
d'homme, E. Banks joue manifestement avec ce qu'elle a choisi de ne pas être et avec les attentes masculines.

Ces recoupements, que j'effectue en tant que lectrice, expliquent que, lorsque Banks interroge ses collègues blanchisseuses sur leur situation familiale, elle s'étonne qu'elles ont fait le choix de se marier sans rien «y gagner » (CC 204). En employant l'expression 'bettering their condition', Banks trahit le fait que sa perception du mariage est celle d'un état favorable à la femme, qui achète ainsi un confort de vie. La révélation de ce que le labeur de ces femmes est choisi par goût et par plaisir conduit à un intéressant constat sur l'émancipation et l'indépendence de ces femmes qui font le choix de travailler audelà du salaire que cet emploi représente :

These laundry-workers were not without their theories concerning woman's emancipation. When they married they had no thoughts of giving up what they termed their 'independence'. They clung to that with the greatest tenacity, and were under the impression that, by earning from ten to twenty shillings a week in a laundry, they were able to hold it fast. In a word, they all seemed 'laundry-struck,' and marriage seemed to mean no more to them than an escape from being called an 'old maid.' (CC 204)

En d'autres termes, les motifs qui conduisent ces femmes à travailler sont les mêmes que ceux qui animent Banks elle-même.

\section{« Il faut éprouver pour savoir »}

La présence physique de Banks dans ses écrits, jugée encombrante et peu pertinente par certains lecteurs, est essentielle à son observation participante. Elle donne à voir avec un certain pathos le décalage entre son corps menu et délicat et les tâches infligées aux servantes et aux blanchisseuses. L'observation participante repose sur l'hypothèse qu' « il faut éprouver pour savoir $»^{27}$. Les activités observées par Banks ne sont pas évaluées par des chiffrages ou en fonction de critères extérieurs, mais à partir de son expérience personnelle, ce qui donne davantage de légitimité à sa critique des conditions de travail imposées : elle déplore les horaires déraisonnables, l'insalubrité des locaux, la pénibilité du métier de servante et le manque d'initimité qui leur est laissé.

Le lecteur constate comment les illusions de puissance et de supériorité de la jeune journaliste qui prétendait révolutionner l'approche des tâches ménagères et ouvrir à sa classe de nouveaux débouchés professionnels sont entièrement prises en défaut. Par un curieux retournement, Banks conquiert le succès médiatique grâce à une écriture de l'échec. Elle témoigne de son épuisement, de son dégoût, de son incapacité et de sa révolte. Elle fait l'expérience de ses limitations : 'I put my head down on the kitchen table and silently wept' (CC 29), 'my head ached for the want of food' (CC 30), 'I was so tired, I could hardly stand' (CC 179). Les places de domestiques et de travailleuses manuelles sont difficiles à obtenir et à conserver. Le corps lui-même s'y refuse, et Banks sera plusieurs fois congédiée avant même d'avoir donné sa démission. Devenue blanchisseuse, dans la continuité de son ressenti ${ }^{28}$, elle regarde autour d'elle et observe que les corps qui l'entourent sont difformes : 'bent shoulders', 'deformity', 'humped' (CC 189). Cette prise de conscience de la dégénérescence ouvrière est explicitement décrite comme une conséquence de son propre état physique, si bien qu'elle est mise sur le compte des conditions de travail et non de l'hérédité. 

pittoresque en même temps que du sentimentalisme, est le pendant féminin de ce que les Victoriens appelaient 'nature's gentleman', elle est noble de cœur et pleine de grâce : 'she looked as though she should have painted pictures or made music for the world, instead of living in and breathing an atmosphere of soap-suds' (CC 168). Boiteuse et légèrement bossue, Janie a une cicatrice sur le front et un doigt écrasé. Son corps a une fonction argumentative forte, car il porte les marques de dix-huit ans d'une existence dure et laborieuse : après une chute sur le pavé lorsqu'elle était bébé, Janie a buté sur un gardefeu, s'est écrasée le doigt en coupant du bois, avant que ses positions au travail lui déforment l'épaule. L'état physique de Banks et les épreuves par lesquelles elle vient de passer la rendent sensible à la façon dont le milieu a modifié le corps de Janie jusqu'à le rendre monstrueux.

deux articles dont Banks notait la futilité dans sa préface sont probablement ceux qui démontrent le mieux comment la journaliste est entièrement transformée par son contact avec la profession : devenue balayeuse, elle se sent prise de sentiments de haine vis-à-vis de ceux, dont elle faisait partie, qui ne récompensent pas les efforts des balayeurs. Devenue vendeuse de fleurs, sa personnalité se mue d'audacieuse et franche en humble et vulnérable, confrontée à cette impitoyable profession et à leurs redoutables praticiennes.

Sans cesse évaluée par ses collègues et maitresses, Banks prend conscience de son insignifiance. Elle découvre qu'elle n'a pas beaucoup d'énergie (CC 196) et qu'elle n'apprend pas facilement ('Hit seemes so 'ard for you to learn things', CC 186). Lorsque, de retour à New-York, elle continua, comme 'yellow journalist' à s'immerger dans des milieux étrangers et peu enviables : 'I had always to carry with me spirits of camphor and smelling-salts, for I was continually feeling ill and faint from the foul odours that assailed me, and there were times when my heart almost stopped beating from fear.' (ANG 214) Sa décision d'être journaliste en deviendrait presque un choix par défaut; telle est la conclusion comique de Campaigns of Curiosity, lorsque Janie, devant les multiples échecs de sa protégée, se voit forcée de réfléchir à lui trouver un autre gagne-pain. Après un long débat où ses collègues blanchisseuses l'envisagent successivement dans des emplois de comptable, d'infirmière et de serveuse, elles ne voient d'autre solution pour elle que de vendre des bonbons (CC 183).

\section{Écriture de soi}

Engagée dans les 'deceptive practices' étudiées par Seth Koven ${ }^{29}$ ('incognitos, investigative journalism, falsified photographs'), avec lesquelles sa pratique autobiographique forme continuité, Banks excelle à se mettre en position d'imposture et possède un style haut en couleurs et en sensations multiples, riche en anecdotes, qui vise à flatter le goût du grand public. Ce que Jean Copans appelle « l'écriture du terrain » par distinction avec «l'écriture de terrain » encourt souvent le rique d'être «terriblement personnalisé et littéraire $»^{30}$. L'écriture de Banks a des qualités littéraires qui expliquent son succès auprès des lecteurs en son temps et aujourd'hui, où ses œuvres conservent un intérêt qui n'est pas seulement documentaire. Mais la place centrale occupée par l'auteur dans ses écrits, et le soin et l'insistance accordés à la textualisation de ses expériences (Autobiography of a Newspaper Girl reprend presque in extenso des épisodes de Campaigns of Curiosity) signalent que cette étape fait partie de sa réflexion sur ce vécu.

Revue Française de Civilisation Britannique, XV-4 | 2010 

troisième chapitre à examiner la continuité qui existe entre l'observation des enfants et des adolescents éduqués librement, celle qu'exerce le futur sociologue sur son milieu d'origine et la pratique de l'observation participante. Il conclut qu'il n'y a pas de solution de continuité entre toutes ces activités d'observation ${ }^{31}$. À quel moment le simple observateur devient-il sociologue? Lorsqu'il analyse sa propre pratique. L'observateur deviendra sociologue lorsqu'il apprendra à s'autoanalyser ${ }^{32}$. Tel est le cas de Banks puisque, de même que William Foot Whyte écrivit pour son compte-rendu d'enquête sur Cornerville à Boston en 1937 un appendice autobiographique décrivant les préliminaires et les conditions matérielles de son enquête (appendice devenu plus célèbre que l'enquête elle-même) ${ }^{33}$, Elizabeth Banks a décrit dans Autobiography of a Newspaper Girl les mêmes incidents et démarches que dans ses Campaigns of Curiosity, mais analysés depuis l'angle de sa propre entrée dans le monde du journalisme. Elle y présente notamment ses nombreux scrupules envers les aspects non-éthiques de son activité. Banks ne semble avoir eu d'autre vie que celle qui l'a conduite à jouer de multiples rôles, mais ses enquêtes sont des étapes dans un parcours personnel : ils lui permettent de conquérir une position difficile de femme journaliste; elle les présente aussi comme des étapes dans sa maturation.

En mettant en scène ces retournements, Banks opère un bouleversement des repères et des idées, un élargissement des sensations qui repousse, selon elle, les limites de son individualité. Dans les dernières pages de son autobiographie, après avoir donné un bref aperçu de ses diverses observations participantes aux États-Unis (dans les 'sweatshops', les prisons, les hôpitaux, la Morgue, des écoles...), elle s'exclame :

'The Way of Life'! Truly I now began to walk in that way. Truly I began to grow. As the days and the weeks went on I could even feel myself growing, growing in grace, growing in charity, putting aside such narrow creeds and prejudices as had been a part of my up-bringing, and were, perhaps, in their place and time, good and wholesome for the girl, but cramping, distorting, warping to the woman. Life ! Life ! - Seething life was all about me! The life of a great city - its riches, its poverty, its sins, its virtues, its sorrows, its joyousness - there it was, and I was in it. This life was no longer like a panorama spread out for me to look at simply, to smile or weep over, and then to turn away my eyes from beholding it. I entered it, and, while I studied, became a part of it, learning how akin was all humanity, and how large a place had environment and circumstance in the making of character and the moulding of destiny. (ANG 215)

Ces belles lignes font état d'un ressenti qui ne s'invente pas. Seth Koven les interprète comme trahissant repli sur soi et frilosité : 'as always she focused on the impact of her slumming on her own subjectivity'34. Au lieu d'aboutir à un propos généralisant sur la société américaine de son époque ou à des révélations inédites, son récit débouche en effet sur une analyse de sa propre pratique de sociologue, où les idées d'immersion et de transformation occupent une place centrale. Ces lignes me semblent curieusement proches d'un passage de Jacques Coenen-Huther dans son introduction à une série d'études réalisées par lui-même sous la forme d'observations participantes dans des domaines très variés.

Franchir le temps et l'espace pour établir des invariants du comportement humain implique que l'éloignement culturel ne constitue pas un obstacle insurmontable. Il faut pouvoir se mettre à la place d'autrui - autrui qui peut être à tout point de vue très lointain - et lui attribuer une logique de comportement dont on puisse percevoir le sens. Ceci passe par une démarche intellectuelle, introspective d'abord, projective ensuite. (...) Une des fonctions de l'observation participante - et ce 
pourrait être sa fonction la plus spécifique - est précisément de favoriser la décentration en élargissant cognitivement le champ des possibles ou, en d'autres termes, en rendant davantage de logiques particulières accessibles à l'entendement. Pratiquée avec prudence, elle prémunit contre les pièges de la projection hâtive. ${ }^{35}$

La subjectivité n'est donc pas un obstacle à la connaissance mais son instrument principal, à condition que celle-ci soit soumise à des conditions changeantes mais non différentes de façon à modifier de façon significative la perception et les impressions. L'observateur-participant ne vise pas un inédit à découvrir, il se méfie des révélations. Ce qui fait la supériorité de l'expérience de Banks sur celles d'autres 'stunt journalists', c'est son choix d'explorer autrement des situations familières et non exotiques et titillantes ${ }^{36}$. Même si sa négociation d'accès au terrain fait apparaitre les difficultés à obtenir les positions convoitées, les rôles joués en Angleterre ne sont pas lointains. Une exception pourrait être faite pour le cas de la riche Américaine cherchant à s'introduire dans la meilleure société anglaise, mais l'article prouva précisément les failles de ce système exclusif et provoqua un renforcement des contrôles exercés sur l'introduction à la cour d'Angleterre. permet d'envisager une situation d'un nouveau point de vue et de modifier sa perception des choses. Le quotidien le plus familier peut ainsi devenir un domaine d'étude, par le seul travail de la subjectivité. Dans la conclusion à sa série de récits d'observation participante, Coenen-Huther témoigne même de la nécessité d'être déjà familier des expériences étudiées :

Il ne suffit pas de se mettre au balcon et de goûter le spectacle de la vie. Il ne suffit pas de se promener le nez au vent en se fiant à son inspiration. (...) comprendre, au sens technique du terme, c'est reconstruire par projection la logique d'un comportement. Cette opération intellectuelle requiert donc l'expérience antérieure, directe ou indirecte, de comportements du même genre. Et les limites de l'expérience dictent les limites de la compréhension possible. Une situation d'interaction ne devient intelligible que sur la base de la participation préalable à d'autres situations présentant les mêmes caractéristiques formelles ou structurelles. ${ }^{37}$

L'écriture de Banks, qui conserve la trace de l'engagement physique de son auteur, permet au lecteur de revivre lui aussi des scènes familières. Que la représentation des faits soit partiale et soumise à de multiples contraintes n'est en rien un obstacle. Comme l'observe Paul Thompson qui utilisa pour écrire son histoire sociale de la période édouardienne des témoignages recueillis sous forme d'entretiens, toute donnée historique est le fruit d'une perception et demande à être interprétée.

These social statistics thus do not represent absolute facts, any more than newspaper reports, private letters, or published biographies. Like recorded interview material, they all represent, either from individual standpoints or aggregated, the social perception of facts; and all are in addition subject to social pressures from the context in which they are obtained. With these forms of evidence, what we receive is social meaning, and it is this which must be evaluated. 38

Elizabeth Banks pratique une micro-sociologie de terrain personnelle dans laquelle différents niveaux de représentation sont imbriqués, mais, comme ce qui précède l'a montré, ces filtres ont l'avantage d'être repérables et ils enrichissent l'analyse. En ce sens, même la littérature joue un rôle essentiel dans la recréation des mentalités pour l'historien ou le civilisationniste, qui ne peuvent pas faire d'observation participante. Analysés synthétiquement, les articles de Banks font apparaître la nécessité du

Revue Française de Civilisation Britannique, XV-4 | 2010 
changement, l'importance du milieu d'où seuls «les génies» peuvent s'extraire (CC 205-206), et le prix accordé par toutes ces femmes à leur "indépendance ", mot-clé qui forme continuité entre sa propre vie et celles qu'elle observe. Les pratiques et les savoirs sont très variables; la réalité sociale décrite par Banks est gouvernée par des rapports intersubjectifs plutôt que par des règles et des lois. Sa méthode lui permet de rendre compte fidèlement de cette dimension des choses.

\section{BIBLIOGRAPHIE}

AUERBACH, Nina. Private Theatricals: The Lives of the Victorians. Harvard UP, 1990.

BANKS, Elizabeth L. The Autobiography of a Newspaper Girl. Londres : Methuen, 1902.

BANKS, Elizabeth L. (intr. M.S. Schriber et A. Zink), Campaigns of Curiosity: Journalistic Adventures of an American Girl in Late Victorian London. The University of Wisconsin Press, 2003.

BERGER, Ronald J. and Richard QUINNEY, Storytelling Sociology: Narrative as Social Inquiry. Boulder, Co, Londres, Lynne Riener, 2005.

COENEN-HUTHER, Jacques. Observation participante et Théorie sociologique. Paris: L'Harmattan, 1995. COPANS, Jean. L'Enquête sociologique de terrain. Armand Collin, 2005.

DOUGLAS, George H. The Golden Age of the Newspaper. Westport, Conn.: Greenwood, 1999.

DOYLE, A. C. The Adventures of Sherlock Holmes. Oxford: OUP, 1993.

HESS, Rémi et Gabriele WEIGAND (dir.). L'Observation participante dans les situations intercultuelles. Paris: Economica, 2006.

HUGHES, Everett C. The Sociological Eye : Selected Papers, New Brunswick/Londres: Transaction, 1993.

KOVEN, Seth. Slumming: Sexual and Social Politics in Victorian London. Princeton UP, 2004.

MOORE, George. Esther Waters, Oxford: OUP, 1999.

PENEFF, Jean. Le Goût de l'observation: Comprendre et Pratiquer l'observation participante en sciences sociales. Paris: La Découverte, 2009.

SCHRIBER, Mary Suzanne. Writing Home: American Women Abroad 1830-1920. Charlottesville/ Londres: UP of Virginia, 1997.

THOMPSON, Paul. The Edwardians: The Remaking of English Society. Londres/New-York: Routledge, 1992.

\section{NOTES}

1. Banks était très préoccupée par ces aspects : voir par exemple le chapitre 'Why I did not become a Salvation Army "lassie"' dans Elizabeth L. BANKS, The Autobiography of a Newspaper Girl, 
Londres: Methuen, 1902. En ligne sur http://www.archive.org. Les références suivantes à cette œuvre seront signalées dans le texte sous la forme ANG.

2. Voir l'introduction de M. S. SCHRIBER et Abbey ZINK in Elizabeth L. BANKS, Campaigns of Curiosity: Journalistic Adventures of an American Girl in Late Victorian London, The University of Wisconsin Press, 2003.

3. Elizabeth Banks se fit au contraire un nom en répondant à l'article de Rudyard Kipling 'On One Side Only' (The Times, 29 nov. 1892) en défendant le point de vue de la jeune femme américaine.

4. Il faut peut-être y voir un désir de se distinguer des expériences sensuelles racontées par The Amateur Casual, frère du journaliste Frederick Greenwood, qui, après une nuit passée dans une workhouse avait raconté ses expériences dans une série d'articles publiés dans The Pall Mall Gazette. Voir Seth KOVEN, Slumming: Sexual and Social Politics in Victorian London, Princeton UP, 2004. Chapitre 1.

5. 'The relation of my experiences, coming at a time when the question of shorter hours and more perfect sanitation for laundries has been brought before Parliament by the Home Secretary, I hope may not be without the effect of calling attention to a class of working girls who stand in great need of a helping hand from the better classes.' Elizabeth L. BANKS (intr. M.S. Schriber et A. Zink), Campaigns of Curiosity: Journalistic Adventures of an American Girl in Late Victorian London,The University of Wisconsin Press, 2003, p. xiv. Les références suivantes à cet ouvrage seront signalées dans le texte sous la forme CC.

6. Seth KOVEN, op. cit., pp. 167-69.

7. Nina AUERBACH, Private Theatricals: The Lives of the Victorians, Harvard UP, 1990.

8. Pour écrire son histoire financière de la City, Ranald C. MICHIE a traité la littérature comme une source importante. Voir Guilty Money: The City of London in Victorian and Edwardian Culture (1815-1914). Londres: Chatto \& Windus, 2009.

9. Dans son autobiographie, Elizabeth BANKS raconte également son expérience de la cueillette des fraises (ANG 99-102).

10. Voir 'L'observation participante' de Georges LAPASSADE dans Remi HESS et Gabriel WEIGAND (dir.), L'observation participante dans les situations interculturelles. Paris : Economica, 2006.

11. Jean PENEFF, Le Goût de l'observation : Comprendre et Pratiquer l'observation participante en sciences sociales, Paris : La Découverte, 2009, pp. 82-91.

12. Jean COPANS, L'Enquête sociologique de terrain, Armand Collin, 2005, p. 35.

13. Jean PENEFF n'hésite même pas à à parler des acteurs comme de praticiens de l'observation participante (op. cit., pp. 90-91).

14. Jean PÉNEFF, op. cit. pp. 120-22, 124, 125.

15. Ibid., p. 88.

16. Ibid., cité p. 90.

17. Idem.

18. Ronald J. BERGER and Richard QUINNEY, Storytelling Sociology: Narrative as Social Inquiry, Boulder, Co, Londres, Lynne Riener, 2005, p. 3.

19. 'She is the only person I encountered in researching this book who unashamedly used the word "slumming" to describe her own activities in London.' Seth KOVEN, op. cit., p. 161.

20. Comme l'explique l'un des grands noms de l'observation participante, Everett HUGHES, la pratique de l'observation participante elle-même génère une variété de rôles sociaux à l'intérieur du groupe étudié : 'The outstanding peculiarity of this method is that the observer, in greater or lesser degree, is caught up in the very web of social interaction which he observes, analyzes, and reports. Even if he observes through a peephole, he plays a role: that of spy. And when he reports his observations made thus he becomes a kind of reformer. If he observes in the role of a member of the group, he may be considered a traitor the moment he reports.' Everett C. HUGHES, The Sociological Eye: Selected Papers, New Brunswick/Londres : Transaction, 1993, p. 505. 
21. Elizabeth Banks explique dans sa biographie les raisons qui motivèrent son refus de se faire passer pour une prostituée ou de mener l'enquête à l'Armée du Salut. 'The very first thing I was asked to do in the line of "yellow" work was to walk along Broadway at midnight and "allow" myself to be arrested and sent to the lock-up as a disreputable character all this in order to bring about a reform in certain laws that were obnoxious to many New Yorkers, and to prove, without a doubt, that a respectable woman walking quietly to her home late at night, was liable to be pounced upon and arrested.' (ANG 205)

22. 'I do not mean that any beggar in the streets of London could earn 700 pounds a year - which is less than my average takings - but I had exceptional advantages in my power of making up, and also in a facility of repartee, which improved by practice and made me quite a recognised character in the City. All day a stream of pennies, varied by silver, poured in upon me, and it was a very bad day in which I failed to take two pounds.' Arthur Conan DOYLE, The Adventures of Sherlock Holmes. Oxford: OUP, 1993, p. 146.

23. Georges LAPASSADE, op. cit., p. 17.

24. Dans Esther Waters (1894), George MOORE donna à voir les préjugés et la discrimination qui s'exerçaient à l'égard des nourrices. George MOORE, Esther Waters, Oxford: OUP, 1999, pp. 140, $175-76,182$.

25. Seth KOVEN, op. cit., p. 5.

26. Dans le chapitre 'When I began to starve', Banks raconte comment elle dut vendre sa machine à écrire et en racheter une à crédit pour pouvoir financer le matériel nécessaire à son séjour d'étude (ANG 72-74).

27. Jean PENEFF, op. cit., p. 10.

28. 'I had every reason to believe that my lungs were of the strongest, and would stand any reasonable amount of carelessness on my part, but I contracted a hacking cough, which, happily, did not last long after I made my final exit from the atmosphere of soap-suds. While engaged in the work, however, I was constantly in terror of getting maimed or killed with the machinery, and so, as I had given a false name and false address to the manageress of the laundry, I wore always in the locket round my neck a thin slip of paper upon which I had written particulars of what was to be done with me should any accident befall me.' (ANG 104-105).

29. Seth KOVEN, op. cit, p. 19.

30. Jean COPANS, op. cit., p. 34 .

31. Jean PENEFF, op. cit., p. 14.

32. Jean PENEFF, op. cit., p. 80.

33. Georges LAPASSADE, op. cit., p. 16.

34. Seth KOVEN, op. cit., p. 148. Voir aussi au sujet du compte-rendu de son expérience de balayeuse de rue : 'we learn a great deal about Banks's feelings' (KOVEN, p. 165). En fait, ce que Koven reproche surtout à Banks, c'est d'avoir ignoré la place de la sexualité dans ses expériences, et de ne jamais se livrer à une réflexion sur les sexes. A la fin de son chapitre, il accorde pourtant à Banks d'avoir pratiqué une certaine forme de sociologie: 'Banks presented her findings largely through descriptions of what she herself did, felt and saw in her various assumed roles. Her method of collecting data and her style in presenting it combined to produce an intensely personal sociology in which the self and the social are purposely intermingled.' (Seth KOVEN, op. cit. p. 169)

35. Jacques COENEN-HUTHER, Observation participante et théorie sociologique, Paris: L'Harmattan, 1995 , p. 15.

36. Tandis que 'The Amateur Casual' se complaisait à décrire le 'weak mutton broth' dans lequel il doit se baigner pour accéder à sa place dans la Workhouse (Seth KOVEN, op. cit., p. 39), Banks épargne à ses lecteurs la description de l'eau du lavabo qu'elle craint de partager avec ses comparses (CC 22).

37. Jacques COENEN-HUTHER, op. cit., p. 175.

38. Paul THOMPSON, The Edwardians: The Remaking of English Society, Londres/New-York: Routledge, 1992, p. xviii-xix. 


\section{RÉSUMÉS}

Dans une série d'articles regroupés sous le titre Campaigns of Curiosity (1894), la journaliste américaine Elizabeth L. Banks étudia la société anglaise de l'intérieur en se faisant passer pour une servante, une blanchisseuse, une balayeuse... Ce texte qui relève à la fois du journalisme d'immersion, de l'autobiographie et du récit de voyages est très écrit et riche en sensations. Il constitue aussi un premier exemple de la méthode de sociologie qualitative appelée observation participante. Banks affronte ses principaux enjeux: la négociation de l'accès au terrain, le décentrement, l'écriture du corps et l'auto-analyse.

In a series of articles collected in Campaigns of Curiosity (1894), the American journalist Elizabeth L. Banks tells how she played the parts of the servant, the launderer, the crossing-sweeper... in order to gain a better knowledge of English society. Part stunt journalism, part autobiography, part travel narrative, the text is also an early case of participant observation, a qualitative research method in sociology. Banks deals with its main aspects: gaining entry into the group, decentering, writing about her experience, analysing her own practice.

\section{AUTEUR}

\section{NATHALIE SAUDO-WELBY}

Université de Picardie 